\title{
Implementation of Venoarterial Extracorporeal Membrane Oxygenation in Nonintubated Patients
}

\author{
Hyeon A Kim, M.D., Young Su Kim, M.D., Yang Hyun Cho, M.D., Ph.D., Wook Sung Kim, M.D., Ph.D., \\ Kiick Sung, M.D., Ph.D., Dong Seop Jeong, M.D., Ph.D. \\ Department of Thoracic and Cardiovascular Surgery, Samsung Medical Center, Sungkyunkwan University School of Medicine, Seoul, Korea
}

\author{
ARTICLE INFO \\ Received June 9, 2020 \\ Revised September 30, 2020 \\ Accepted October 5, 2020 \\ Corresponding author \\ Yang Hyun Cho \\ Tel 82-2-3410-2213 \\ Fax 82-2-3410-0089 \\ E-mail mdcho95@gmail.com \\ ORCID \\ https://orcid.org/0000-0003-1685-3641
}

\begin{abstract}
Background: Although extracorporeal membrane oxygenation (ECMO) is generally performed percutaneously, the technology is deployed under sedation and necessitates endotracheal intubation. However, in some patients, the use of venoarterial (VA) ECMO without intubation may be beneficial. Herein, we describe our experiences with VA ECMO performed without prior endotracheal intubation.

Methods: A total of 783 patients treated with VA ECMO at a single center between January 2013 and July 2018 were reviewed retrospectively. We included patients who underwent successful VA ECMO implementation without prior endotracheal intubation, and excluded those who were younger than 18 years, had ongoing cardiopulmonary resuscitation status, and had poor quality of the vessels needed for percutaneous cannulation. The primary study outcome was in-hospital survival.

Results: In total, 50 patients were included in this study, 94\% of whom showed cardiogenic shock. The mean age of the study participants was $56.3 \pm 14.5$ years. The median VA ECMO support time was 7 days (range, 2-13 days). Twenty-one patients (42\%) did not receive ventilator care during the VA ECMO support period, while 29 patients (58\%) progressed to intubation after VA ECMO implementation. The rates of survival at discharge and weaning success were $82 \%(n=41)$ and $92 \%(n=46)$, respectively, and $80 \%(n=40)$ of patients presented good Glasgow-Pittsburgh Cerebral Performance Categories scores at discharge.

Conclusion: Even in patients with cardiogenic shock, percutaneous VA ECMO can be introduced safely without prior endotracheal intubation by an experienced care team. The application of nonintubated VA ECMO might be a feasible strategy in selected cases.
\end{abstract}

Keywords: Extracorporeal membrane oxygenation, Intubation, Endotracheal

\section{Introduction}

Extracorporeal membrane oxygenation (ECMO) is widely used for pulmonary or cardiopulmonary support of unstable patients. Recently, a dramatic increase in the frequency of ECMO use has occurred; catheterization can now be performed at the bedside or in a catheterization laboratory, and medical personnel have more experience with the technology. Usually, ECMO deployment is completed percutaneously using Seldinger's technique under local anesthesia [1].

Given the increasing number of ECMO cases and growing concerns about the outcomes of long-term mechanical ventilator care $[2,3]$, several studies have attempted to ascertain whether prior endotracheal intubation or mechanical ventilator support is necessary for the success of ECMO treatment. Investigations of ECMO in awake patients have mainly involved venovenous (VV) ECMO during spontaneous breathing. Notably, most of these studies were conducted in patients with respiratory failure from acute respiratory distress syndrome or patients waiting for lung transplantation [4-6]. Separately, although few cases were included, some studies have also explored awake venoarterial (VA) ECMO in patients in cardiogenic shock [7-10].

There are definitive benefits associated with prior endotracheal intubation before VA ECMO implementation, in- 


\section{January 2013-July 2018 in SMC patients with} ECMO implementation $(n=783)$

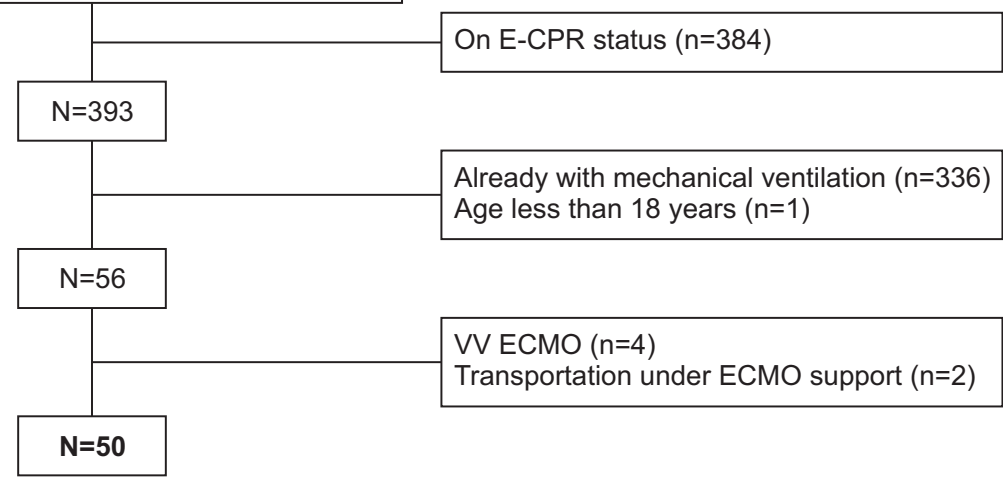

Fig. 1. Patient selection. From January 2013 to July 2018; in total, 783 patients were treated with ECMO implementation at SMC. SMC, Samsung Medical Center; ECMO, extracorporeal membrane oxygenation; E-CPR, extracorporeal cardiopulmonary resuscitation; $\mathrm{VV}$, venovenous. cluding the establishment of optimal conditions for the delivery of therapy with relatively well-controlled vital signs, the absence of concerns regarding respiratory failure, and avoidance of patient agitation or movement. However, in some patients experiencing cardiogenic shock, the preconditioning stage required for endotracheal intubation might deteriorate the vital signs by reducing native sympathetic tone and may be a time-consuming aspect of the procedure [4]. If the etiology of shock is obviously cardiogenic and quick decision-making by an experienced care team is feasible, the straightforward implementation of VA ECMO without prior intubation might be of greater benefit to the patient. Based on this hypothesis, herein, we describe our experiences with VA ECMO implementation preceding endotracheal intubation. Notably, we seek to distinguish this protocol from previous "awake ECMO" protocols as specifically referring to nonintubated VA ECMO implementation.

\section{Methods}

\section{Study population and procedures}

We retrospectively reviewed the records of 783 patients who were treated with ECMO at Samsung Medical Center, Seoul, Republic of Korea between January 2013 and July 2018. We included patients who underwent successful percutaneous VA ECMO introduction without prior endotracheal intubation. Nonintubated VA ECMO deployment was performed in selected patients with spontaneous breathing and peripheral vessels deemed suitable for the necessary percutaneous access. A multidisciplinary team composed of cardiac surgeons, cardiologists, and critical care specialists made the decision to pursue nonintubated VA ECMO implementation, weighing the risks and bene- fits of nonintubated VA ECMO use in each case, albeit rapidly given the urgent context. The contraindications for nonintubated VA ECMO implementation included ongoing cardiopulmonary resuscitation (CPR) status and poor quality of the vessels needed for percutaneous cannulation. Patients who were already intubated, who required VV ECMO implementation, who were younger than 18 years of age, and/or who were transported from another hospital under ECMO support were excluded from our study analysis (Fig. 1). The implementation of VA ECMO was performed in the catheterization laboratory, intensive care unit (ICU), or operating room (OR). Candidates were placed under local anesthesia (2\% lidocaine, subcutaneous injection) together with intravenous pain-control medications (fentanyl or pethidine). Spontaneous breathing was facilitated with oxygen administered by a mask. All procedures were performed percutaneously using Seldinger's technique; ultrasound-guided cannulation and fluoroscopy were used in the catheterization laboratory and blind or ultrasound-guided procedures were performed in the ICU or OR. Immediately after VA ECMO introduction, a Doppler sensor was applied to the distal part of the arterial cannulation site and a distal perfusion catheter was added if needed.

This study was approved by the Institutional Review Board of Samsung Medical Center (IRB approval no., 2018-09-099-002). The requirement for informed consent was waived because this was a retrospective study. All personal patient data, along with clinical, laboratory, and outcomes data, were collected from patients' medical records.

\section{Definitions and outcomes}

Nonintubated VA ECMO is defined as the introduction of VA ECMO without prior endotracheal intubation at the 
Table 1. Demographics of patients

\begin{tabular}{|c|c|c|c|c|}
\hline Characteristic & Total $(n=50)$ & Nonintubation $(n=21)$ & Intubation ( $\mathrm{n}=29)$ & p-value \\
\hline Age (yr) & $56.3 \pm 14.5$ & $61 \pm 13.5$ & $52.9 \pm 14.5$ & 0.04 \\
\hline Male & $34(68)$ & $14(67)$ & $20(69)$ & 0.86 \\
\hline Body mass index $\left(\mathrm{kg} / \mathrm{m}^{2}\right)$ & $22.4 \pm 3.6$ & $23 \pm 3.5$ & $21 \pm 3.7$ & 0.06 \\
\hline \multicolumn{5}{|l|}{ Comorbidities } \\
\hline Hypertension & $22(44)$ & $9(42)$ & $13(45)$ & 0.89 \\
\hline Diabetes & $26(52)$ & $12(57)$ & $14(48)$ & 0.53 \\
\hline Malignancy & $5(10)$ & $2(9)$ & $3(10)$ & $>0.99$ \\
\hline Chronic kidney disease & $3(6)$ & 0 & $3(10)$ & 0.25 \\
\hline \multicolumn{5}{|l|}{ Previous medical history } \\
\hline Cerebrovascular disease & $5(10)$ & $1(4)$ & $4(14)$ & 0.38 \\
\hline Myocardial infarction & $7(14)$ & $2(9)$ & $5(17)$ & 0.68 \\
\hline Percutaneous coronary intervention & $9(18)$ & $4(19)$ & $5(17)$ & $>0.99$ \\
\hline Coronary artery bypass grafting & $5(10)$ & $3(14)$ & $2(4)$ & 0.63 \\
\hline
\end{tabular}

Values are presented as mean \pm standard deviation or number (\%).

Table 2. The location of VA ECMO implementation and other variables

\begin{tabular}{|c|c|c|c|c|}
\hline Variable & Total $(n=50)$ & Nonintubation $(n=21)$ & Intubation $(\mathrm{n}=29)$ & p-value \\
\hline \multicolumn{5}{|l|}{ VA ECMO implementation } \\
\hline Location of implementation & & & & 0.68 \\
\hline Intensive care unit & $4(8)$ & $3(14)$ & $1(3)$ & \\
\hline Catheterization lab & $44(88)$ & $18(86)$ & $26(90)$ & \\
\hline Operating room & $2(4)$ & 0 & $2(7)$ & \\
\hline Reasons for implementation & & & & $>0.99$ \\
\hline Cardiogenic shock & $47(94)$ & $19(90)$ & $28(97)$ & \\
\hline Obstructive shock & $2(4)$ & $2(9)$ & 0 & \\
\hline For the operation & $1(2)$ & 0 & $1(3)$ & \\
\hline VA ECMO duration (day) & $7(2-13)$ & $5.2(1.4-13.9)$ & $8.1(2.7-17.8)$ & 0.40 \\
\hline \multicolumn{5}{|c|}{ Events during VA ECMO implementation } \\
\hline Stroke events & $4(8)$ & 0 & $4(14)$ & 0.1 \\
\hline Limb ischemia & $2(4)$ & 0 & $2(7)$ & 0.3 \\
\hline \multicolumn{5}{|l|}{ Bleeding events } \\
\hline VA ECMO site & $7(14)$ & $3(14)$ & $4(14)$ & 0.6 \\
\hline Gastrointestinal bleeding & $2(4)$ & 0 & $2(7)$ & 0.3 \\
\hline \multicolumn{5}{|l|}{ Intubation duration } \\
\hline$<24 \mathrm{hr}$ & & & $17(58.6)$ & \\
\hline $1 \leq$ day $\leq 7$ & & & $6(20.7)$ & \\
\hline$>7$ day & & & $6(20.7)$ & \\
\hline
\end{tabular}

Values are presented as number (\%) or median (range).

VA ECMO, venoarterial extracorporeal membrane oxygenation.

time of VA ECMO implementation. "Awake ECMO" is a relatively broad concept that defines a status in which mechanical ventilation support is removed during the maintenance period regardless of whether it was present at the time of VA ECMO or VV ECMO implementation. Therefore, we distinguished our patient pool from those receiving awake ECMO by using the term "nonintubated VA ECMO."

The primary study outcome was survival to hospital discharge and the secondary outcomes were successful VA
ECMO weaning and neurological status at the time of discharge. The Glasgow-Pittsburgh Cerebral Performance Categories (CPC) scale was used to measure patients' neurological status at discharge. CPC scores of 1 or 2 points were regarded as suggesting good neurological outcomes.

All included patients who underwent nonintubated VA ECMO implementation were divided into 2 groups: those who eventually required intubation during the VA ECMO maintenance period (intubation group) and those who did not (nonintubation group). In addition, patients were strat- 
Table 3. Details of various origins of cardiogenic shock

\begin{tabular}{lccc}
\hline \multicolumn{1}{c}{ Details } & $\begin{array}{c}\text { Acute coronary } \\
\text { syndrome }(\mathrm{n}=16)\end{array}$ & $\begin{array}{c}\text { Acute decompensated } \\
\text { heart failure }(\mathrm{n}=31)\end{array}$ & Others $(\mathrm{n}=3)$ \\
\hline STEMI & 7 & - & - \\
Non-STEMI & 7 & - & - \\
Stable angina & 1 & - & - \\
Unstable angina & 1 & 5 & - \\
Myocarditis & - & 16 & - \\
Dilated CMP & - & 6 & - \\
Heart failure, unspecified & - & 3 & - \\
Ischemic CMP & - & 1 & - \\
Stress-induced CMP & - & - & 1 \\
Others & & - & 1 \\
$\quad$ Idiopathic pulmonary fibrosis & - & - & 1 \\
\hline Pulmonary embolism & - & - & \\
\hline Prophylactic before general anesthesia & - & & \\
\hline
\end{tabular}

Values are presented as number of patients.

STEMI, ST-elevation myocardial infarction; CMP, cardiomyopathy.

ified into 3 groups-acute coronary syndrome (ACS), acute decompensated heart failure (HF), and other-according to the etiology underlying VA ECMO implementation. The primary and secondary outcomes were also compared according to these etiologies.

\section{Statistical analysis}

Mean values and standard deviations were used to evaluate patients' basic characteristics and outcomes. The Fisher exact test and the chi-square test were used to compare primary and secondary endpoints between the groups. The values for survival at discharge and CPC scores at discharge were calculated for all patients. Kaplan-Meier curves were constructed to determine the estimated survival and was drawn based on the last follow-up date rather than the discharge date. The log-rank test was performed to compare the results of each group. Statistical analyses were executed using SAS ver. 9.4 (SAS Institute Inc., Cary, NC, USA). A p-value of less than 0.05 was considered to indicate statistical significance.

\section{Results}

\section{Patient characteristics}

A total of 50 patients were enrolled in this study. The mean age of the study participants was $56.3 \pm 14.5$ years and $34(68 \%)$ were men. The mean body mass index of patients was $22.4 \pm 3.6 \mathrm{~kg} / \mathrm{m}^{2}$ and a majority of the patients were either normal body weight or overweight (Table 1). Forty-four

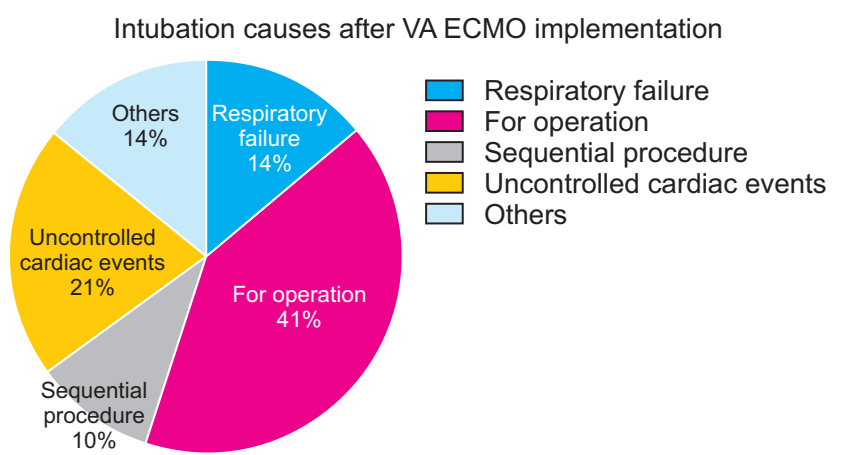

Fig. 2. Reasons for endotracheal intubation after VA ECMO implementation. In patients with nonintubated VA ECMO implementation, 29 patients underwent endotracheal intubation for the reasons shown above. Twelve patients underwent intubation for an operation (9 for heart transplantation, left ventricular assisted device implantation, or coronary artery bypass grafting and 3 for other operations), 6 for uncontrolled cardiac events, 4 due to respiratory failure, and 3 for sequential procedures (septal puncture procedures in the catheterization laboratory). VA ECMO, venoarterial extracorporeal membrane oxygenation.

patients (88\%) had VA ECMO initiated in the catheterization laboratory. The cause of VA ECMO implementation was mainly cardiogenic shock $(\mathrm{n}=47)$, followed by obstructive shock $(n=2)$, and as part of a preoperative preparation protocol $(\mathrm{n}=1)$ (Table 2). Among patients with cardiogenic shock, 16 patients had ACS and 31 patients had acute decompensated HF. The specific origins of cardiogenic shock are described in detail in Table 3. The median duration of VA ECMO maintenance was 7 days (range, 2-13 days). During the VA ECMO maintenance period, 29 patients (58\%; intubation group) required endotracheal intubation 
Table 4. Primary and secondary outcomes in subgroups (intubation and nonintubation groups)

\begin{tabular}{lcccc}
\hline \multicolumn{1}{c}{ Variable } & Total $(\mathrm{n}=50)$ & Nonintubation $(\mathrm{n}=21)$ & Intubation $(\mathrm{n}=29)$ & $\mathrm{p}$-value \\
\hline Survival at discharge & $41(82)$ & $20(95.2)$ & $21(72.4)$ & 0.06 \\
Weaning success & $46(92)$ & $21(100)$ & $25(86.2)$ & 0.12 \\
Good CPC score at discharge & $40(80)$ & $20(95.2)$ & $20(69)$ & 0.03 \\
\hline
\end{tabular}

Values are presented as number (\%).

CPC, Glasgow-Pittsburgh Cerebral Performance Categories.

Table 5. Primary and secondary outcomes in subgroups in subgroups (acute coronary syndrome and acute decompensated heart failure groups)

\begin{tabular}{lccr}
\hline \multicolumn{1}{c}{ Variable } & $\begin{array}{c}\text { Acute coronary syndrome } \\
(\mathrm{n}=16)\end{array}$ & $\begin{array}{c}\text { Acute decompensated heart } \\
\text { failure }(\mathrm{n}=31)\end{array}$ & $\mathrm{p}$-value \\
\hline Survival at discharge & $14(87.5)$ & $24(77.4)$ & 0.69 \\
Weaning success & $16(100)$ & $27(87.1)$ & 0.28 \\
Good CPC at discharge & $13(81.3)$ & $24(77.4)$ & $>0.99$ \\
Intubation during VA ECMO implement & $9(56.3)$ & $10(32.3)$ & 0.10 \\
\hline
\end{tabular}

Values are presented as number (\%).

CPC, Glasgow-Pittsburgh Cerebral Performance Categories; VA ECMO, venoarterial extracorporeal membrane oxygenation.

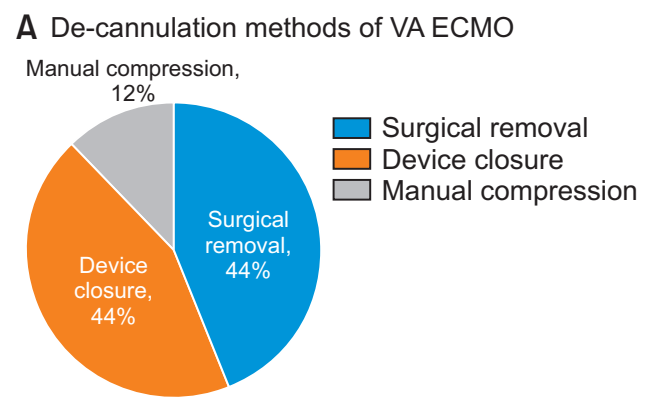

B De-cannulation methods of VA ECMO in the intubation group

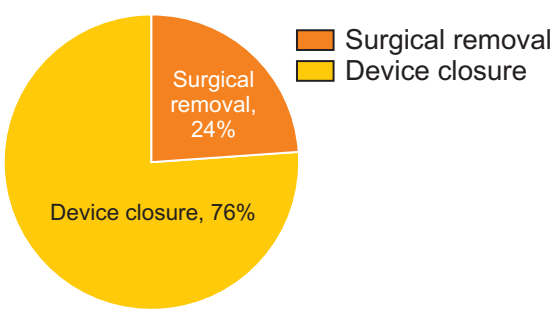

Fig. 3. De-cannulation methods of VA ECMO. (A) Of the 50 total patients, 46 patients achieved weaning success from VA ECMO. De-cannulation of VA ECMO was performed by surgical removal in 20 patients (44\%), device closure in 20 patients (44\%), and manual compression in 6 patients (12\%). (B) In the intubation group, de-cannulation of VA ECMO was performed by device closure in 16 patients $(76 \%)$. VA ECMO, venoarterial extracorporeal membrane oxygenation.

with mechanical ventilator support. The causes of intubation were for operation in 12 patients, respiratory failure in 4 patients, persistent cardiac events after VA ECMO implementation in 6 patients, and as sequential treatment immediately after VA ECMO implementation in 3 patients (Fig. 2).

\section{Outcomes}

The rate of survival at discharge was $82 \%$, the weaning success rate among all patients was $92 \%(\mathrm{n}=46)$, and $80 \%$ $(n=40)$ of patients presented good CPC scores at the time of discharge. Notably, the rates of survival at discharge (95.2\% versus $72.4 \%, \mathrm{p}=0.06$ ) and the VA ECMO weaning success rate $(100 \%$ versus $86.2 \%, p=0.12)$ were not significantly different between the nonintubation and intubation groups. However, the proportion of patients with good
CPC scores at discharge (95.2\% versus $69 \%, \mathrm{p}=0.03)$ was higher in the nonintubation group (Table 4). The primary and secondary outcomes were also compared according to the etiology underlying VA ECMO implementation; among the 47 patients with cardiogenic shock, 16 patients had ACS and 31 patients had acute decompensated HF. The rate of survival at discharge was not significantly different between patients with ACS and with acute decompensated HF ( $87.5 \%$ versus $77.4 \%, p=0.69)$. The rates of weaning success $(100 \%$ versus $87.1 \%, \mathrm{p}=0.28)$ and good CPC score at discharge $(81.3 \%$ versus $77.4 \%, \mathrm{p}>0.99)$ were also not significantly different between the 2 groups (Table 5). Fig. 3 shows the de-cannulation methods for VA ECMO. Of the 50 patients, 46 achieved weaning success from VA ECMO. De-cannulation of VA ECMO was performed by surgical removal in 20 patients (44\%), device closure in 20 patients 

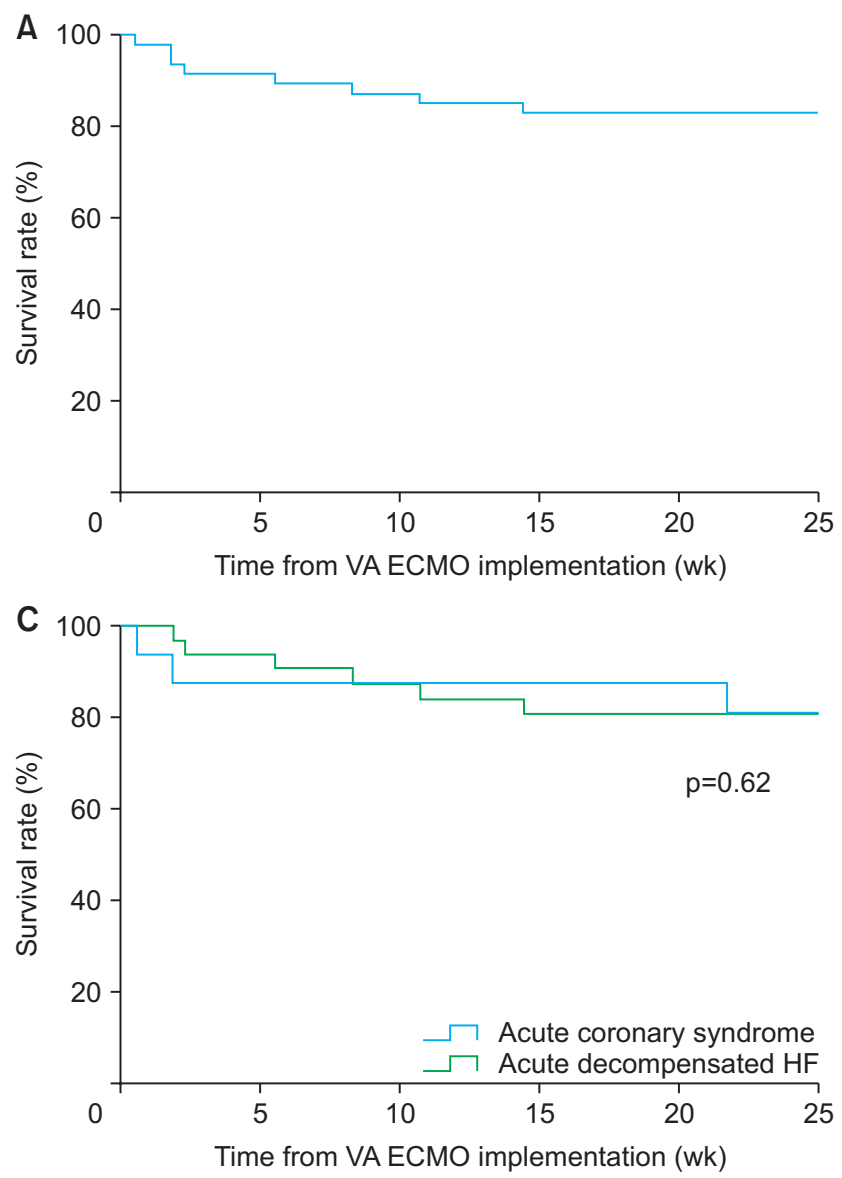

(44\%), and manual compression in 6 patients (12\%). The 6-month survival analysis using the log-rank test for the nonintubation and intubation groups showed no significant difference (85.7\% versus $75.9 \%, \mathrm{p}=0.71$ ) (Fig. 4B). There was also no significant difference in 6-month survival between the ACS and acute decompensated HF groups (81.3\% versus $80.6 \%, \mathrm{p}=0.62)$ (Fig. $4 \mathrm{C}$ ).

\section{Discussion}

In most cases, patients who require VA ECMO implementation present in a state of shock or with marginal vital signs. In this situation, preparation for endotracheal intubation, including intravenous injection of sufficient sedatives or ensuring optimal positioning, may deteriorate the barely maintained vital signs of some patients. In selected patients, if there are no particular concerns in the plan to implement VA ECMO, nonintubated VA ECMO might be better for maintaining intrinsic sympathetic tone and reducing the total procedure time by eliminating the time needed for preconditioning. In patients presenting with

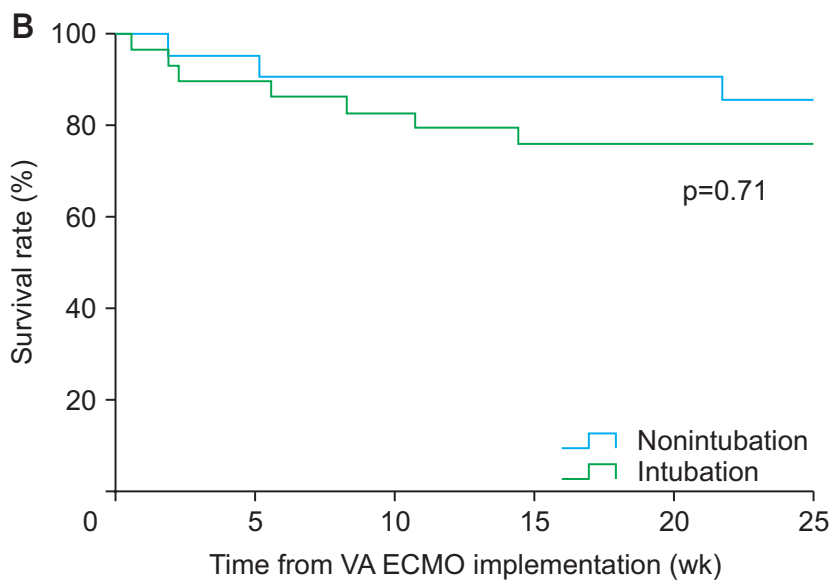

Fig. 4. Kaplan-Meier curves for estimated 6-month (24-week) survival. (A) Analysis of all patients, (B) analysis according to progression to intubation, and (C) results of ACS and acute decompensated HF subgroup analysis. VA ECMO, venoarterial extracorporeal membrane oxygenation; ACS, acute coronary syndrome; HF, heart failure.

cardiogenic shock, no specific characteristics are considered to be indications for intubation and assisted ventilation, although Levy et al. [11] noted that mechanical ventilation should be more carefully administered in certain situations. The 2016 European Society of Cardiology guidelines also state that noninvasive ventilation should be considered preferable over mechanical ventilation in patients with HF [12]. There are also well-known drawbacks of mechanical ventilation following endotracheal intubation. In particular, long-term mechanical ventilation is a double-edged sword that may lead to significant active inflammation and pulmonary damage, which can be risk factors for acute lung injury $[2,3]$.

Importantly, there are definitive benefits of performing endotracheal intubation before VA ECMO implementation, including ensuring optimal conditions with relatively well-controlled vital signs, reducing concerns about respiratory failure, and eliminating the potential for patient agitation or movement. However, with an experienced team, prior endotracheal intubation is not necessary in the context of VA ECMO implementation. 
Several studies have assessed patients receiving VV ECMO without mechanical ventilator support as ECMO has become increasingly common and amid concerns about the effects of long-term mechanical ventilator care. Olsson et al. [13] reported good results after applying awake VV ECMO treatment in 5 lung-transplantation candidates. Since then, awake VV ECMO treatment has been mostly used in this patient population $[4,14,15]$. In many cases, good results were reported, but these included nonintubated VV ECMO cases as well as extubated patients receiving ECMO support.

To date, very few cases of awake VA ECMO have been reported, especially those only receiving nonintubated VA ECMO implementation. Deng et al. [7] reported their experience pursuing awake VA ECMO in patients with perioperative acute HF, noting many benefits of awake VA EMO treatment, such as reduced pulmonary complications, improved rehabilitation, and less muscle atrophy and venous thrombosis. Several reports have also demonstrated good results associated with nonintubated VA ECMO use in patients with ACS [8,9]. Mori et al. [10] reported good results after adopting nonintubated VA ECMO as a bridge to ventricular-assisted device implementation.

At Samsung Medical Center, patients who recently underwent VA ECMO implementation had a weaning success rate of $70 \%$ and a survival rate at discharge ranging between $55 \%$ and $59 \%$. In this study, the outcomes of patients who underwent nonintubated VA ECMO implementation were not inferior to the overall outcomes mentioned above. This is because patients who received VA ECMO with ongoing CPR were excluded from this study; the outcomes of these patients are usually worse than those of patients without emergent CPR. Furthermore, in this study, $88 \%$ of patients underwent planned or early VA ECMO implementation in the catheterization laboratory.

Although the results may vary depending on many factors, such as patient characteristics, primary disease, disease severity, and underlying diseases, this study reveals that outcomes were generally worse in the intubation group. Surgery, which was the major cause of intubation, was performed for heart transplantation in many cases or for conversion to central ECMO to facilitate long-term maintenance. Patients who were expected to fail weaning from ECMO inevitably underwent surgery, which consequently affected their outcomes. In addition, patients with respiratory problems or uncontrolled cardiac events had a worse prognosis than those without these characteristics. The logrank test results of the Kaplan-Meier curves showed no significant differences (Fig. 4B).
In addition to the comparison of intubation and nonintubation groups, a comparison was conducted according to the etiology of cardiogenic shock. Among 47 patients in cardiogenic shock, 16 had ACS and 31 had acute decompensated HF. There were also 3 patients with other etiologies: one was hemodynamically unstable with pulmonary embolism, another was a candidate for pulmonary transplantation with hemodynamic instability, and the third was a patient at high risk for adverse outcomes when using general anesthesia for surgery. There were no statistically significant differences in the primary and secondary outcomes between these groups (Table 5) and the log-rank test results of the Kaplan-Meier curves showed no significant difference between the ACS and acute decompensated HF groups (Fig. 4C).

In some contexts, nonintubated VA ECMO implementation could seem like a reckless process in patients in cardiogenic shock. However, there are some conditions that made the conduct of this study reasonable. First, almost all of the enrolled patients had been selected for admittance to or were already in the cardiac ICU or the cardiac surgery ICU. Of course, all patients showed suspected cardiogenic shock, which was diagnosed by bedside echocardiography, electrocardiography, or elevated cardiac enzyme level, and were monitored by cardiologists or cardiac surgeons. Second, there were adequate resources at hand, including monitoring systems, easy access to the catheterization laboratory, and the availability of experienced health care staff who could perform endotracheal intubation or other conventional life support treatments if needed.

Importantly, this study had several limitations. First, it was a retrospective study and the data were collected through a review of patient medical records, which may introduce systematic errors that might have affected outcomes, including differences in patient characteristics between groups. However, common sense suggests that patients with worse conditions would eventually be intubated, and there were few meaningful differences between the 2 groups. Second, the study population was not large, which can weaken its statistical accuracy. Studies involving a large number of subjects have not been published to date; further investigations should include larger groups of patients.

In conclusion, this study is meaningful in that it focused solely on patients receiving nonintubated VA ECMO at a time when data on this modality are lacking. Further, we attempted to distinguish and clarify the nature of nonintubated VA ECMO from previous awake ECMO treatments. In this study, nonintubated VA ECMO implementation 
showed comparable results to VA ECMO implementation in other cases. Nonintubated VA ECMO implementation might be feasible and presents an opportunity to reconsider the stereotypical VA ECMO implementation. Nonintubated VA ECMO implementation could be a new strategy that may be implemented carefully in selected patients in cardiogenic shock by an experienced team.

\section{Conflict of interest}

No potential conflict of interest relevant to this article was reported.

\section{ORCID}

Hyeon A Kim: https://orcid.org/0000-0002-0648-948X

Young Su Kim: https://orcid.org/0000-0003-2923-3732

Yang Hyun Cho: https://orcid.org/0000-0003-1685-3641

Wook Sung Kim: https://orcid.org/0000-0001-7808-3385

Kiick Sung: https://orcid.org/0000-0003-0768-9587

Dong Seop Jeong: https://orcid.org/0000-0002-6947-8403

\section{References}

1. Guglin M, Zucker MJ, Bazan VM, et al. Venoarterial ECMO for adults: JACC Scientific Expert Panel. J Am Coll Cardiol 2019;73: 698-716

2. Strieter RM, Lynch JP 3rd. Complications in the ventilated patient. Clin Chest Med 1988;9:127-39.

3. Pierson DJ. Complications associated with mechanical ventilation. Crit Care Clin 1990;6:711-24

4. Fuehner T, Kuehn C, Hadem J, et al. Extracorporeal membrane oxygenation in awake patients as bridge to lung transplantation. Am J Respir Crit Care Med 2012;185:763-8.

5. Langer T, Santini A, Bottino N, et al. "Awake" extracorporeal membrane oxygenation (ECMO): pathophysiology, technical considerations, and clinical pioneering. Crit Care 2016;20:150.
6. Hoopes CW, Kukreja J, Golden J, Davenport DL, Diaz-Guzman E, Zwischenberger JB. Extracorporeal membrane oxygenation as a bridge to pulmonary transplantation. J Thorac Cardiovasc Surg 2013; 145:862-7.

7. Deng L, Xia Q, Chi C, Hu G. Awake veno-arterial extracorporeal membrane oxygenation in patients with perioperative period acute heart failure in cardiac surgery. J Thorac Dis 2020;12:2179-87.

8. Liu S, Ravandi A, Kass M, Elbarouni B. A case of awake percutaneous extracorporeal membrane oxygenation for high-risk percutaneous coronary intervention. Cureus 2017;9:e1191.

9. Van Houte J, Donker DW, Wagenaar LJ, Slootweg AP, Kirkels JH, van Dijk D. Non-intubated recovery from refractory cardiogenic shock on percutaneous VA-extracorporeal membrane oxygenation. Neth Heart J 2015;23:386-8.

10. Mori M, McCloskey G, Geirsson A, et al. Improving outcomes in INTERMACS category 1 patients with pre-LVAD, awake venous-arterial extracorporeal membrane oxygenation support. ASAIO J 2019; 65:819-26

11. Levy B, Bastien O, Karim B, et al. Experts' recommendations for the management of adult patients with cardiogenic shock. Ann Intensive Care 2015;5:52.

12. Ponikowski P, Voors AA, Anker SD, et al. 2016 ESC guidelines for the diagnosis and treatment of acute and chronic heart failure: the Task Force for the diagnosis and treatment of acute and chronic heart failure of the European Society of Cardiology (ESC) developed with the special contribution of the Heart Failure Association (HFA) of the ESC. Eur Heart J 2016;37:2129-200.

13. Olsson KM, Simon A, Strueber M, et al. Extracorporeal membrane oxygenation in nonintubated patients as bridge to lung transplantation. Am J Transplant 2010;10:2173-8.

14. Hayes D Jr, Kukreja J, Tobias JD, Ballard HO, Hoopes CW. Ambulatory venovenous extracorporeal respiratory support as a bridge for cystic fibrosis patients to emergent lung transplantation. J Cyst Fibros 2012;11:40-5.

15. Biscotti M, Gannon WD, Agerstrand C, et al. Awake extracorporeal membrane oxygenation as bridge to lung transplantation: a 9-year experience. Ann Thorac Surg 2017;104:412-9. 